\title{
Special issue on source water risk control
}

\author{
Shupei Cheng $\cdot$ Tim Ford
}

Accepted: 20 May 2009/Published online: 7 June 2009

(C) Springer Science+Business Media, LLC 2009

The First International Workshop on Environmental Health and Pollution Control, EHPC2006, was held at Nanjing University, PR China, in October 2006. The event attracted environmental scientists from five countries to present their research and engage in a rich discussion about critical environmental health issues. This first meeting focused on four key areas: Environmental health and biotechnology; environmental engineering and technology; predictive models; and environmental planning and management. The meeting identified the vast scale of environmental problems that need to be addressed and was notable in the extraordinary level of enthusiasm amongst participants to make progress. The second international workshop, EHPC2008, on which this special edition of ecotoxicology is based, was a logical progression from the groundwork done at the first workshop.

Over the past century, China has faced rapid growth in both urbanization and industrialization and has submitted the China National Environment \& Health Action Plan (2007-2015) on November 21 of 2007 to begin to address the elevated levels of water and air pollution that threaten the health of both rural and urban populations (China National Environment and Health Action Plan 2007). It is both encouraging and enlightening to see a new cadre of scientists beginning to address these issues and this special

\footnotetext{
S. Cheng

State Key Laboratory of Pollution Control and Resources Reuse, School of the Environment at Nanjing University,

210093 Nanjing, China

T. Ford ( $\square)$

University of New England, 11 Hills Beach Road,

Biddeford, ME 04005, USA

e-mail: tford@une.edu
}

edition is specifically produced to support their efforts and promote their future contributions to the field.

We have organized this edition into five general sections that reflect specific areas of interest amongst the workshop attendees, these areas are in:

1. Contaminant concentrations and genetic markers

2. Use of ecotoxicological models (algae, cell lines, earthworms and bacteria)

3. Use of mouse models to assess toxicity of source and drinking water

4. Degradation

5. Ecological risk

\section{Contaminant concentrations and genetic markers}

The first three papers look at ways to detect contaminants in the environment. The first paper provides a description of the many organic pollutants that are detectable in fish and zoobenthos sampled from the Yangtze River. It is important to note that detection of contaminant chemicals in environmental media alone does not imply a human health risk, as minimal or no information is obtained on the bioavailability of the chemicals, nor the affects of exposure to complex mixtures (Monosson 2005). The second paper describes a molecular approach to monitoring environmental contaminants, in this case presence of specific antibiotic resistance genes in river and lake water and in drinking water treatment bioreactors. Measurement of genetic markers of exposure to contaminants provides an indication of current or past exposure to bioavailable molecules, in this case antibiotics, and is an approach increasingly used to understand microbial ecology in contaminated environments (Bouskill et al. 2007; Lachmayr 
et al. 2009). One paper in this edition of ecotoxicology looks at method development for detection of pathogens, in this case, Giardia lamblia, a common contaminant of source and drinking waters. The authors compare different protocols for direct DNA extraction from G. lamblia seeded surface water samples, in order to optimize real-time PCR detection of this pathogen.

\section{Use of ecotoxicological models (algae, cell lines, earthworms and bacteria)}

The field of ecotoxicology depends on the development of relatively rapid and simple tools to detect potential pollution problems in any given body of water. Although mice have been traditionally used in toxicological studies for hundreds of years (Shayne and Gad 2006), their use is limited for field applications, requires access to a vivarium and strict adherences to animal care and use practices. These limitations do not apply to the use of models described in this section, namely algae, cell lines, earthworms and bacteria. Although these models have an even more tenuous link to human health than mouse models, they may be good indicators of ecosystem health. In the first paper in this section, the utility of the comet assay in providing a sensitive marker of genotoxic effects of organic pollutants from a drinking water source is demonstrated. Subsequent papers show that (1) specific antibiotics and antioxidants inhibit microalgal growth and cause cell membrane damage, (2) that reporter gene assays may be used to test the endocrine disrupting effects of Yangtze River water, and (3) that the oxidative stress response in the earthworm may be a useful indicator of exposure to contaminant chemicals such as brominated flame retardants. Each of these papers contributes new approaches or applications of ecotoxicological tools that can be readily utilized to monitor ecosystem health of surface waters.

The final paper in this section explores the use of microbial communities as potential markers of environmental contamination. The authors use phospholipid fatty acid profiles as indicators of a changing microbial community structure in response to different organics at different concentrations.

\section{Use of mouse models to assess toxicity of source and drinking water}

In recognition of the problem of estimating human health risk based on contaminant concentrations and ecotoxicological models, both in terms of the effects of bioavailable contaminants at very low concentrations and the cumulative effects of exposure to multiple contaminants, the next series of papers focus on toxicity of source waters using mouse models. The first paper in this section provides an analysis of organic compounds and trace elements in source water from the Yangtze River, providing the water quality background for the subsequent papers.

These four papers provide compelling evidence that Nanjing source and drinking water may cause liver toxicity, may contain carcinogens and may potentially damage the male reproductive system. Of course, caution must be taken in extrapolating clinical relevance to health effects in mouse models (Muruganandan and Sinal 2008). However, these studies do provide a strong indication that source and drinking waters may be of concern to human health and that contaminant mixtures, as well as individual chemicals, should be considered in any human health risk assessment.

\section{Degradation}

Studying presence of contaminants and toxicity/ecotoxicity of a water body are the first steps in environmental health and pollution control. However, the ultimate goals are mitigation and remediation. Many contaminants are difficult to remove from the environment by physical and chemical processes, or the technologies are prohibitively expensive. Bioremediation has been long thought of as a potential solution for environmental contamination, particularly where the predominant toxic chemicals are organic (Young and Young 1995). The two papers in this edition that address degradation of organic pollutants take the approach of examining efficacy of fusant strains in degradation of PAHs. In the first paper, one fusant strain appears more effective than its parental strains at removing PAHs from Yangtze River source water, and in the second paper, the same fusant strain is effective in enhancing degradation rates of a specific $\mathrm{PAH}$, benzo(a)pyrene. Both papers conclude that this fusant strain could potentially be applied in drinking water treatment to reduce health risks from exposure to PAHs. With any engineered organisms, further studies are necessary to examine longevity and survivability of these fusant strains in the environment, but this remains an exciting area of research with as yet untapped promise for pollution control.

\section{Ecological risk}

The three final papers address the topic of ecological risk, health diagnosis of a water body and approaches towards establishing an early warning system. The question that these papers pose is how do you evaluate the risk from contaminated water sources and translate that information into an effective response. The first paper assesses risk in relation to concentrations of seven heavy metals based on 
assignment of an established risk assessment code that provides an indication of bioavailable fractions of these metals. The code allows the authors to assign risk, from "Low" to "Very High", for Luan River sediments that could be used to prioritize remediation programs. The second paper constructs indices of health of the Beihuan water system in Beijing that allows the authors to categorize lakes and rivers as healthy, unhealthy or critical, with a specific health ranking and an estimate of how easy it might be to restore the water body to a "healthy" condition, again providing information for resource management decisions. The final paper takes a very different approach and looks at the potential structure and technical requirements of an early warning system for the Taihu Lake Basin. The paper describes a potential process for moving from real time environmental data collection through the modeling process to a risk management system. The authors acknowledge that considerable research challenges remain - in, for example, reducing uncertainty, model calibration and verificationbefore effective early warning systems can be established.

\section{Framework for the future}

Through collaboration and an open discussion of remediation and pollution control technologies, we can make much more rapid progress than we will ever achieve in isolation. Pollution should concern all of us as effects are global, with chronic disease suspected to be linked with pollution on the opposite side of the globe. For example, indigenous peoples of the arctic have been studied over a number of years to evaluate the health consequences of exposure to persistent organochlorines and toxic metals in their diet (Van Oostdam et al. 2005). These contaminants may be generated at low latitudes, yet are transported to high latitudes through the process of global distillation (Joseph et al. 2008). In addition to the potential for chronic disease, studies of the Nunavik population in Northern Quebec have linked prenatal exposure to organochlorines with childhood acute respiratory infections (Dallaire et al. 2006).

These international conferences on environmental health and pollution control, EHPC 2006 and EHPC 2008, are truly a way to establish productive collaborations, foster exchange of faculty and students, and effectively begin to address the pollution crisis. These series of papers can contribute to an overall scheme for risk management that is simplistically demonstrated by Fig. 1. Understanding each step of the process will provide environmental professionals with the tools to begin to reduce exposure through both contaminant mitigation and source control.
Fig. 1 Scheme for risk management

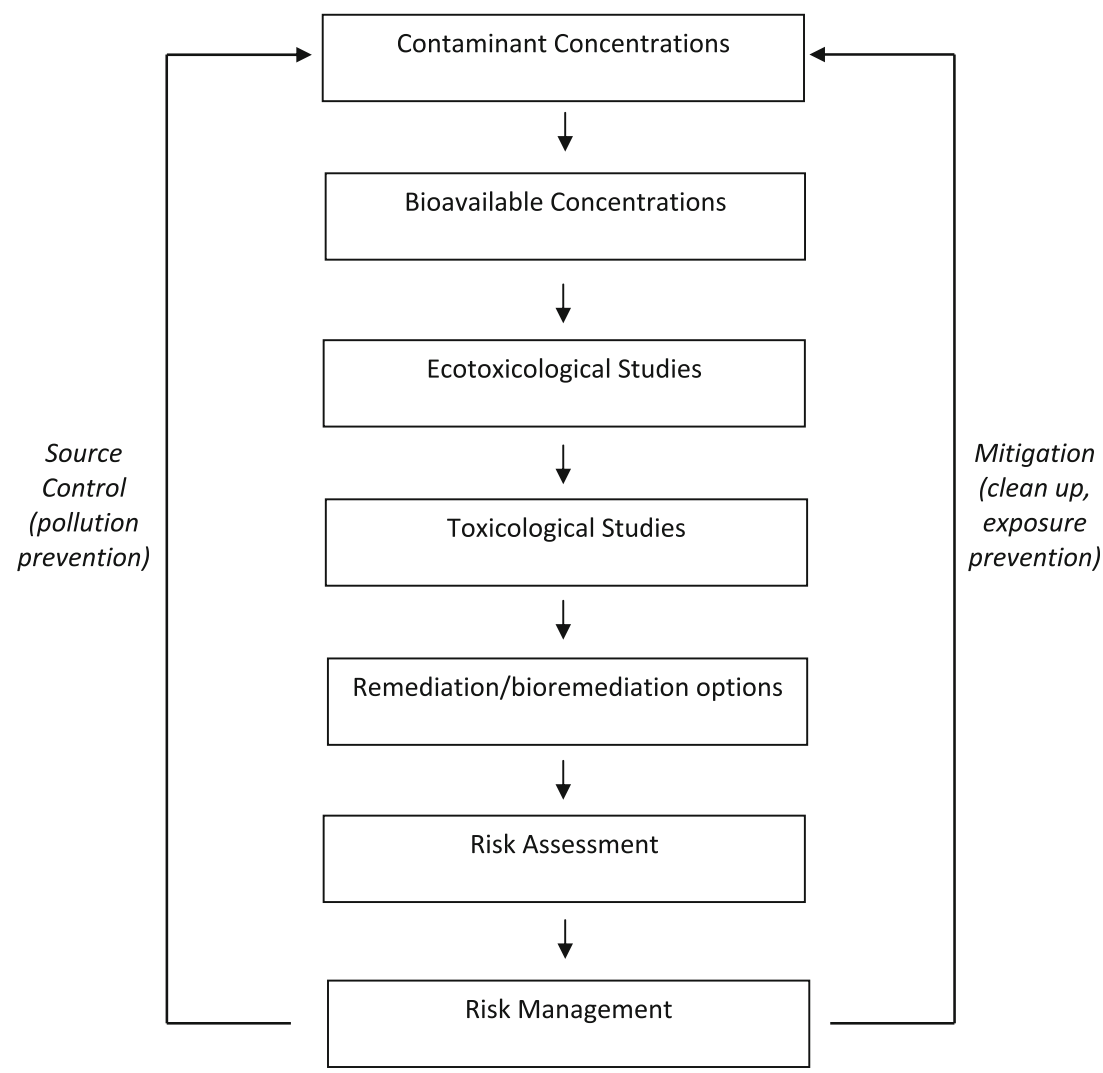




\section{References}

Bouskill NJ, Barnhart E, Galloway T, Handy R, Ford T (2007) Quantification of changing Pseudomonas aeruginosa SodA, $\mathrm{HtpX}$ and Mt gene abundance in response to trace metal toxicity: a potential biomarker of ecosystem health. FEMS Microbiol Ecol 60:276-287. doi:10.1111/j.1574-6941.2007.00296.x

China National Environment and Health Action Plan (2007-2015) http://www.wpro.who.int/NR/rdonlyres/13A55C9E-9383-47E89F24-F23FDA48F066/0/NEHAPfinaledition.pdf 2007; 24 pp

Dallaire F, Dewailly E, Vézina C, Muckle G, Weber J-P, Bruneau S, Ayotte P (2006) Effect of prenatal exposure to polychlorinated biphenyls on incidence of acute respiratory infections in preschool Inuit children. Environ Health Perspect 114:1301-1305

Joseph R, McConnell JR, Edwards R (2008) Coal burning leaves toxic heavy metal legacy in the Arctic. Proc Natl Acad Sci U S A 105:12140-12144. doi:10.1073/pnas.0803564105.PMCID:PMC 2529165

Lachmayr KL, Kerkhof LJ, DiRienzo G, Cavanaugh CM, Ford TE (2009) Quantifying non-specific TEM $\beta$-lactamase genes in a wastewater stream. Appl Environ Microbiol 75:203-211. doi: 10.1128/AEM.01254-08

Monosson E (2005) Chemical mixtures: considering the evolution of toxicology and chemical assessment. Environ Health Perspect 113:383-390

Muruganandan S, Sinal CJ (2008) Mice as clinically relevant models for the study of Cytochrome P450-dependent metabolism. Clin Pharmacol Ther 83:818-828. doi:10.1038/clpt.2008.50

Shayne C, Gad SC (eds) (2006) Animal models in toxicology. Taylor \& Francis, London

Van Oostdam J, Donaldson SG, Feeley M, Arnold D, Ayotte P, Bondy G, Chan L, Dewaily E, Furgal CM, Kuhnlein H, Loring E, Muckle G, Myles E, Receveur O, Tracy B, Gill U, Kalhok S (2005) Human health implications of environmental contaminants in Arctic Canada: a review. Sci Total Environ 351352:165-246. doi:10.1016/j.scitotenv.2005.03.034

Young LY, Young CE (eds) (1995) Microbial transformation and degradation of toxic organic chemicals. Wiley, New York 